\title{
Análise de Redes Ópticas em Anéis Hierárquicos
}

\author{
M. M. O. Silva, M. H. M. Paiva, F. R. de Souza, G. Lavagnoli, M. O. Lima, \\ C. R. L. Francês, J. C. A. W Costa, R. T. R. Almeida, E. Oliveira e M. E. V. Segatto
}

\begin{abstract}
Resumo-Esse artigo apresenta uma metodologia para o projeto completo de redes ópticas em anéis hierárquicos. Partindo da matriz de demanda de tráfego, foram analisadas todas as etapas de projeto: o projeto da topologia virtual, a alocação de comprimentos de onda e roteamento de tráfego e a análise de desempenho da rede com o simulador de redes ópticas OWns. São apresentados os resultados para o desempenho de redes de 60 nós em função de características de sua topologia física.
\end{abstract}

Palavras-Chave-Redes ópticas, projeto de redes, anéis hierárquicos.

Abstract-This paper presents a methodology for the complete design of optical networks based on hierarchical rings. Starting from the matrix of traffic demand, all the stages of design had been analyzed: the design of the virtual topology, the routing and wavelengths assignment of traffic and the analysis of performance of the network with the simulator OWns. The results for the performance of networks of 60 nodes as a function of the characteristics of its physical topology are presented.

Keywords-Optical networks, network design, hierarchical rings.

\section{INTRODUÇÃO}

O projeto realista de uma rede de telecomunicações é um problema complexo, dada a grande quantidade de variáveis tecnológicas e econômicas que devem ser levadas em consideração durante todas as suas etapas.

As redes ópticas, anteriormente formadas por enlaces ópticos ponto-a-ponto com multiplexação de comprimentos de onda Wavelength Division Multiplexing (WDM), evoluíram para redes com roteamento de tráfego por comprimentos de onda, onde há possibilidade de definição de caminhos ópticos interligando diretamente quaisquer pares de nós [14].

A topologia formada pelos caminhos ópticos, ou topologia virtual, é consideravelmente independente da topologia física, pois esta última está explicitamente definida pelo início e fim dos enlaces de fibra óptica. Por outro lado, a topologia virtual é flexível, dada a capacidade de comutação de comprimentos de onda já disponível em nós de uma rede óptica, com a utilização de Optical Cross-Connects (OXCs) e Optical Add-Drop Multiplexers (OADMs) [14]. Com os recursos de comutação de comprimentos de onda, uma rede óptica pode ser reconfigurada dinamicamente para atender a mudanças nas demandas de tráfego e também para restaurar a topologia lógica em caso de falhas.

M. M. O. Silva, M. H. M. Paiva, F. R. de Souza, G. Lavagnoli, M. O. Lima e M. E. V. Segatto: Laboratório de Telecomunicações, Programa de Pós-Graduação em Engenharia Elétrica; E. Oliveira: Departamento de Ciência da Informação, Universidade Federal do Espírito Santo, Vitória, Brasil. R. T. R. Almeida: Coordenadoria de Eletro-Eletrônica, Centro Federal de Educação Tecnológica do Espírito Santo, Vitória, Brasil. C. R. L. Francês e J. C. A. W Costa: Departamento de Engenharia Elétrica e de Computação, Universidade Federal do Pará, Belém, Pará. E-mail: segatto@ele.ufes.br. Esse trabalho foi parcialmente financiado pela CAPES e pelo CNPq.
A multiplexação de comprimentos de onda possibilita o uso racional da grande largura de banda disponível em uma fibra óptica com a utilização simultânea de vários canais, ou caminhos ópticos, podendo cada canal operar de maneira totalmente independente dos outros, tanto com relação ao protocolo, quanto com relação à codificação, modulação ou taxa de transmissão de dados. A capacidade de transmissão agregada de um enlace óptico com múltiplas fibras ópticas, cada uma com diversos caminhos ópticos associados a diferentes comprimentos de onda, costuma ser normalmente bastante superior à capacidade de processamento de roteadores eletrônicos convencionais, definindo o gargalo eletrônico em redes ópticas de transporte de dados. Portanto, o projeto da infra-estrutura de redes ópticas deve levar em conta tanto seus recursos ópticos como também os recursos eletrônicos, eliminando tanto os gargalos quanto o superdimensionamento, definindo uma topologia virtual que possibilite obter o melhor desempenho possível quanto ao transporte de dados.

Além da otimização da infra-estrutura da rede óptica, um bom projeto de rede deve proporcionar mecanismos de proteção contra falhas. Basicamente existem duas categorias de tratamento do tráfego em caso de falha [14]:

- Restauração baseada em redirecionamento de tráfego em malha e;

- Anéis com capacidades redundantes e comutação óptica automática.

A restauração em malha utiliza a comutação eletrônica de pacotes de dados, e o redirecionamento do tráfego se dá em torno do ponto da falha [14]. Este mecanismo de proteção normalmente é implementado pelos protocolos de transporte de pacotes de dados, sendo seu desempenho severamente limitado para aplicação em redes de alta velocidade, devido ao tempo de regeneração ser consideravelmente alto. Já em anéis redundantes, ou Self Healing Rings (SHRs), existem sempre caminhos alternativos por onde o tráfego pode escoar sem grandes perdas, pois o tempo de restauração em caso de falha é muito pequeno, devido à simplicidade da topologia em anel e do mecanismo de restauração. Neste caso, como sempre existem anéis redundantes, redireciona-se o tráfego para o anel de proteção quando ocorre falha no anel principal, bastando apenas comutar os comprimentos de onda atingidos pela falha. Devido a estas características, a topologia física em anel é ainda a mais utilizada em redes ópticas, principalmente em redes SDH/SONET, justamente pela simplicidade e eficiência do mecanismo de restauração. Por este motivo, adotamos como objeto de estudo deste trabalho as redes ópticas com topologias em anéis hierárquicos com capacidade redundante, ou Hierarchical Self Healing Ring (HSHR), uma variação da topologia SHR. 
Como ilustrado em [15], [12], o número de nós em um SHR é limitado pela capacidade dos enlaces e pelo número de saltos entre os nós origem e destino. Considerando estes fatores, Shi e Fonseka [15] propuseram uma arquitetura de rede constituída por anéis hierárquicos. A proposta da arquitetura HSHR consiste em vários SHRs em uma configuração hierárquica, onde SHRs de nível inferior interconectam-se através de um SHR de um nível superior, como mostrado na Figura 1, na seção seguinte, que trata da descrição da topologia HSHR.

Nosso trabalho tem como objetivo apresentar uma metodologia de projeto para redes ópticas com topologias em anéis hierárquicos. Partindo da definição do número de nós da rede e das demandas máximas de tráfego entre cada par de nós, utilizaremos um algoritmo para escolher uma topologia lógica que apresente um bom valor para o congestionamento; definiremos uma estratégia de roteamento de tráfego para a rede; e realizaremos testes de desempenho com o auxílio do software Optical WDM Network Simulator (OWns) [17].

O restante do artigo está dividido da seguinte forma: na Seção II são descritas as redes ópticas em anéis hierárquicos, e a Seção III faz uma breve descrição do OWns. A metodologia de projeto que adotamos é apresentada na Seção IV. Os resultados do caso de estudo estão na Seção V, e na Seção VI são apresentadas as conclusões.

\section{Redes Ópticas em ANÉIS Hierárquicos}

Uma rede óptica em anéis hierárquicos, ou rede HSHR, consiste em conjuntos de nós interligados em anel e um ou mais anéis de hierarquia superior interligando esses conjuntos. A Figura 1 mostra uma rede hipotética de 12 nós e duas hierarquias, sendo $H_{0}$ a hierarquia inferior e $H_{1}$ a hierarquia superior. Todo o tráfego transmitido entre nós pertencentes a um mesmo anel de hierarquia inferior, ou tráfego intra-anel, é roteado internamente na hierarquia inferior, e somente o tráfego transmitido entre nós pertencentes a anéis de hierarquia inferior diferentes, ou tráfego inter-anéis, passa pela hierarquia superior [7]. Dessa forma, o gerenciamento da rede pode ficar concentrado em um número menor de nós, diminuindo o tráfego de informações de controle pela rede. Os nós que pertencem a duas hierarquias simultaneamente são chamados super-nós, e são responsáveis pelo roteamento de tráfego interanéis. Como exemplo, podemos analisar algumas requisições de tráfego. Suponha inicialmente uma requisição do nó 1 para o 4 da rede hierárquica da Figura 1. O tráfego pode ser roteado no sentido horário passando pelos nós 11 e 6 até chegar ao nó 4, ou no sentido anti-horário, passando pelo nó 3 até chegar ao 4. A escolha do sentido de giro no anel irá influenciar na ocupação dos recursos ópticos da rede (ex: número de comprimentos de onda e quantidade de largura de banda) e eletrônicos (ex: número de transceptores e quantidade de processamento em roteadores) e, conseqüentemente, no congestionamento, definido como a quantidade de tráfego designada ao caminho óptico mais solicitado [14]. Note que todo o tráfego da requisição do nó 1 para o nó 4 foi roteado no interior de um anel da hierarquia $H_{0}$.

Vamos supor agora uma requisição de tráfego do nó 2 para o nó 12. Note que os dois nós pertencem a anéis diferentes de

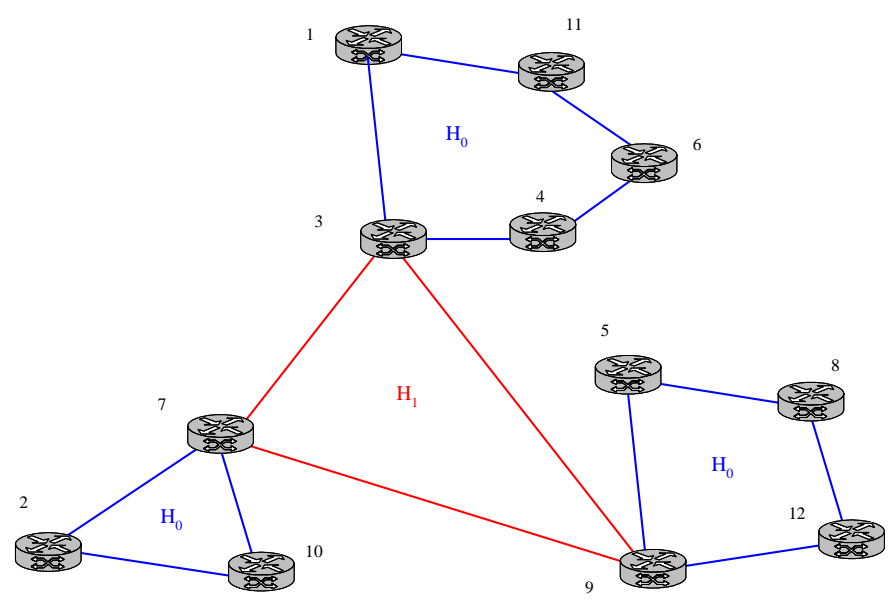

Fig. 1. Um exemplo de rede em anéis hierárquicos: 12 nós e duas hierarquias. $H_{0}$ representa a hierarquia inferior e $H_{1}$ a superior

$H_{0}$. Nesse caso, o tráfego originado no nó 2 deve ser roteado para o super-nó do mesmo anel (nó 7), que por sua vez deve retransmitir o tráfego para o super-nó do outro anel (nó 9), passando assim por um anel de hierarquia superior. $\mathrm{O}$ segundo super-nó fica então encarregado de rotear o tráfego para o nó 12, destino final do tráfego.

\section{O Ambiente de SimulaçÃo OWnS}

O software OWns é uma extensão do Network Simulator (NS-2) [2], que é um software simulador de redes largamente difundido no meio acadêmico. Ambos são gratuitos e código aberto. O núcleo do OWns é implementado em $\mathrm{C}++$ para aproveitar os recursos de modelagem peculiares a essa linguagem. A interface com o operador da simulação é desenvolvida em Object-oriented Tool Command Language (OTcl) por ser uma linguagem interpretada e adequada para construção de scripts. Com relação à arquitetura do OWns, pode-se estabelecer um paralelo com as redes WDM, pois as topologias física e lógica do WDM são mapeadas em camadas física e lógica no OWns.

A camada física é responsável por implementar as características dos nós e enlaces presentes em uma topologia bem como definir os mecanismos de transmissão de pacotes. Em paralelo, a camada lógica é composta pelos módulos de roteamento e atribuição de comprimentos de onda, que em conjunto criam e mantêm a topologia virtual com a função de desempenhar o RWA. A Figura 2 apresenta os principais componentes desta arquitetura e sua organização [11]. O módulo Wavelength Assignment (WA) é responsável por gerenciar a atribuição dos comprimentos de onda, estabelecer os caminhos ópticos e construir a topologia virtual. Por padrão é usado o algoritmo first-fit [2] na atribuição dos comprimentos de onda. Nesse contexto, o OWns permite configurar os nós da rede com ou sem a capacidade de conversão de comprimento de onda. O módulo de roteamento é usado para definir as rotas necessárias para o estabelecimento dos caminhos ópticos. Para isso, o algoritmo fixed-alternate shortest path é usado como padrão. Cabe ressaltar que as rotas construídas seguem 


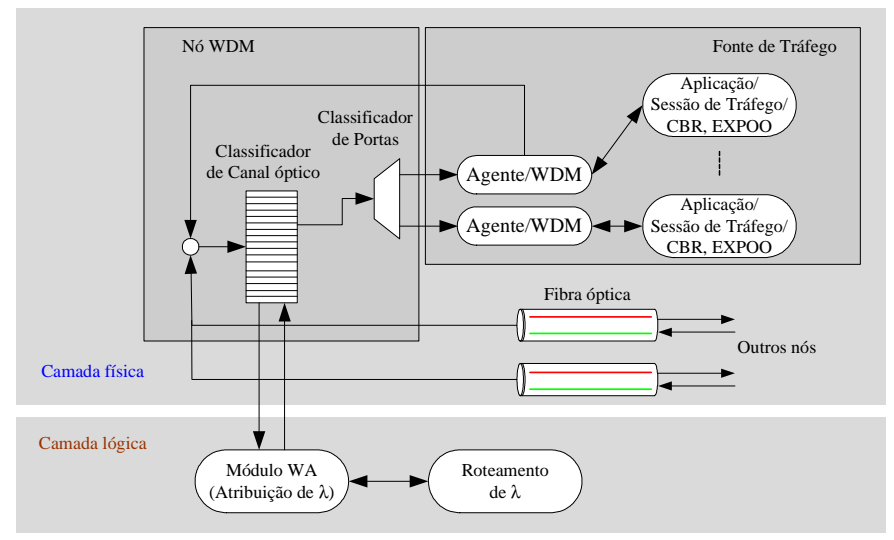

Fig. 2. Arquitetura do OWns.

a seqüência de enlaces físicos presentes na topologia da rede descrita.

Em complemento, os nós WDM são compostos por um classificador de porta e um classificador de caminho óptico. O classificador de porta realiza a função de demultiplexação dos pacotes endereçados ao nó, entregando-os aos respectivos processos. O classificador de caminho óptico interage com o módulo WA para estabelecer os canais ópticos utilizados pelas sessões de tráfego. Nesse sentido, os nós de origem de uma sessão de tráfego solicitam ao módulo WA um canal óptico e, em resposta, o módulo WA estabelece um caminho óptico entre origem e destino reservando recursos e configurando as tabelas de roteamento dos nós intermediários. Os nós intermediários, portanto, apenas consultam a tabela de roteamento para encaminhar os pacotes para o enlace de saída adequado.

Entretanto, durante o processo de definição do caminho óptico, é possível que não haja comprimentos de onda suficientes para atender a demanda da sessão de tráfego solicitada. Nesse caso, a requisição é bloqueada. Assim que recursos alocados por outras sessões forem liberados, a requisição bloqueada poderá ser atendida. Outro fator que acarreta o bloqueio é a falha no processo de conversão entre comprimentos de onda.

Em geral, os enlaces de fibras ópticas são representados no OWns por conexões bidirecionais full duplex. O número de comprimentos de onda e a largura de banda total são parâmetros configurados em cada enlace da rede.

Sobre as estruturas física e lógica descritas são criadas as fontes de tráfego. Elas são responsáveis por gerar carga com características específicas de acordo com o perfil de fluxo desejado. Cada sessão de tráfego pode ser configurada por meio dos parâmetros de taxa média de chegada de sessão (msar - mean session arrival rate) e tempo médio de retenção do tráfego ( $m$ sht - mean session holding time). Também pode ser configurada a distribuição do tempo entre chegadas de pacotes.

Por fim, todos os dados gerados na simulação podem ser armazenados em arquivos de saída em variados formatos. Tais arquivos são usados como entrada para ferramentas de animação gráfica e análise de resultados.

\section{Metodologia de Projeto de Redes Ópticas em ANÉIS HIERÁRQUICOS}

Tradicionalmente, o projeto de redes ópticas é dividido em dois sub-problemas [14]: o projeto da topologia lógica, conhecido como Virtual Topology Design (VTD), e o roteamento e a alocação de comprimentos de onda, ou Routing and Wavelength Assignment (RWA). Com relação à complexidade, ambos sub-problemas são classificados na literatura como NP-árduos [14], [4]. Em geral cada um deles é resolvido independentemente do outro, ou seja, a solução do RWA não leva em consideração os resultados obtidos na solução do VTD, e vice-versa. Posteriormente, as soluções dos dois subproblemas são reunidas, a fim de obter-se a solução geral.

A literatura apresenta uma vasta referência na solução do VTD, seja utilizando programação inteira mista [14], [4], [19], [18], [6] ou métodos heurísticos [14], [9], [8].

O objetivo principal do VTD é, dados uma matriz de demanda de tráfego e um conjunto de restrições, determinar um conjunto de caminhos ópticos que possibilitem que o roteamento de tráfego seja feito de forma a otimizar uma determinada métrica, como o congestionamento [14] ou o processamento de tráfego na rede [4].

O RWA também é resolvido com o auxílio de técnicas de programação inteira mista ou utilizando heurísticas, sendo normalmente modelado como um problema de coloração de grafos [14], [8]. O problema consiste em implementar a topologia virtual em uma dada topologia física com o número mínimo de comprimentos de onda possível.

Soluções para o VTD considerando topologias HSHR podem ser encontradas na literatura para métodos exatos [15], [12], [16], híbridos [9] e heurísticos [7], [13].

No entanto, as técnicas descritas anteriormente buscam soluções exatas ou ótimas para o VTD e para o RWA com um alto custo computacional, e não existe a garantia de que uma solução ótima para o VTD associada a uma solução ótima para o RWA produzam uma solução ótima para o problema como um todo.

O presente trabalho busca elaborar uma metodologia de projeto de redes ópticas em anéis hierárquicos que seja simples e retorne uma solução completa. Com esse intuito, são usados algoritmos de VTD e RWA mais simples, porém capazes de produzir soluções que atendam às restrições de projeto e aos requisitos de desempenho.

Assim, para o VTD é adotado um algoritmo de busca guloso que, dados um conjunto de $\mathrm{N}$ nós com sua localização e uma matriz de tráfego $\Lambda$ com as demandas máximas entre cada par de nós, determina um conjunto de caminhos ópticos em forma de anéis dispostos de forma hierárquica que minimiza o congestionamento da rede. Dentre as diversas soluções encontradas é escolhida aquela com menor valor de congestionamento. Fixado N, esse processo pode ser repetido várias vezes, apenas modificando a quantidade de anéis em $H_{0}$. As melhores topologias obtidas são confrontadas posteriormente para se chegar a uma escolha que melhor atenda ao contexto.

O algoritmo de VTD busca a solução em um subconjunto do total de topologias possíveis. O número de elementos desse subconjunto pode ser definido com base nas análises 
estatísticas apresentadas em [10]. Esse estudo mostra que, em redes ópticas baseadas em anéis hierárquicos, os valores encontrados para o congestionamento ficam concentrados num pequeno intervalo em torno de $0,5 * M$, supondo $\mathrm{M}$ o congestionamento máximo, sugerindo que o comportamento das topologias em função do congestionamento é semelhante ao de uma Distribuição Normal. Sendo assim, mais da metade das topologias geradas apresentam valores muito próximos para o congestionamento, o que justifica a busca em um subconjunto do total de topologias possíveis.

A topologia virtual fornecida pelo processo de VTD é tomada também como topologia física. Essa simplificação é possível devido a características inerentes às redes em anéis hierárquicos [7], [13].

Para definir as rotas necessárias ao estabelecimento dos caminhos ópticos, é usado o algoritmo fixed-alternate shortest path. Este algoritmo realiza cálculos preliminares para encontrar múltiplas rotas entre cada par de nós origem/destino. Os resultados são armazenados na tabela de roteamento de cada um dos nós, em ordem crescente de distância até o destino, e a cada solicitação de sessão de tráfego entre um par de nós, a tabela de roteamento será consultada e a rota com menor distância que esteja disponível será selecionada.

Para atribuição dos comprimentos de onda, é empregado o algoritmo first-fit. Assim, o primeiro comprimento de onda da lista que puder atender a solicitação do caminho óptico será alocado. Ambos os algoritmos são disponibilizados pelo software OWns.

Com o objetivo de comparar o desempenho das redes, as melhores topologias obtidas no processo de VTD são analisadas no OWns. O script é elaborado obedecendo às decisões de projeto, dado que parâmetros da rede como: número de comprimentos de onda, capacidade de transmissão, nós com habilidade para conversão de comprimentos de onda, uso ou não de rotas alternativas no processo de roteamento, entre outros, devem ser fixados. Cabe destacar que todos os parâmetros configurados no script devem ser mantidos para a simulação de todas as topologias, de forma a permitir uma comparação efetiva entre elas.

Configurada a rede, o passo seguinte é destinado ao estabelecimento do tráfego. Para isso, a mesma matriz de demanda $\Lambda$ usada no processo de VTD determina qual o tráfego máximo entre cada par de nós. Diz-se que os valores da matriz são máximos, pois para cada topologia são realizadas simulações com $100 \%, 80 \%, 60 \%, 40 \%$ e $20 \%$ da carga informada.

A matriz de demanda indica qual o volume de dados que necessita ser transmitido por unidade de tempo. Em complemento, também deve ser configurada a distribuição de chegada desses dados para serem transmitidos. São várias as possibilidades, tais como CBR (Constant Bit Rate), Exponencial, Pareto, Erlang, sendo a escolha de um entre esses uma decisão de projeto. A etapa que segue à configuração das fontes de tráfego é o escalonamento dos eventos. Evento, nesse sentido, significa o estabelecimento da comunicação entre um par de nós durante um determinado período de tempo. Os instantes de início e fim de cada evento são estabelecidos randomicamente.

\section{RESUlTADOS}

Para estudo de caso escolhemos redes de 60 nós. A matriz de demanda de tráfego $\Lambda$ foi gerada de forma aleatória com valores de 0,1 e 4 unidades de tráfego, todos com igual probabilidade de ocorrência.

Quanto à configuração das topologias em anéis hierárquicos, consideramos redes com duas hierarquias de anéis, nas quais todos os anéis da hierarquia inferior tem o mesmo número de nós, e cada anel da mesma tem apenas um super-nó.

Com os critérios acima, podemos formar redes com 3, 4, 5, 6, 10, 12 ou 15 anéis na hierarquia inferior (ou super-nós). Para cada uma dessas classes, o algoritmo de VTD gerou aleatoriamente 100000 topologias, calculou o congestionamento de cada uma delas e escolheu a que apresentou congestionamento mínimo. O desempenho das sete topologias resultantes desse processo foi analisado com auxílio do software OWns.

Para a análise no OWns foi adotado o seguinte cenário: os anéis pertencentes à hierarquia $H_{0}$ possuem capacidade máxima de 2,5 Gbps para cada enlace, divididos em 4 tributários de $622 \mathrm{Mbps}$. A hierarquia superior $\left(H_{1}\right)$ possui capacidade para $10 \mathrm{Gbps}$ por enlace divididos em tributários de 2,5 Gbps. Esse procedimento é similar ao utilizado na backbone RNP [1]. Os valores de demanda de tráfego adotados seguiram a mesma proporção, normalizando-se tanto as demandas quanto as capacidades em função da capacidade mínima da rede.

Uma vez escolhida a topologia virtual e definida a estratégia de alocação de recursos, passa-se à configuração das fontes de tráfego. Nessa etapa, são definidas as requisições de tráfego na rede. Caso a demanda seja zero entre dois nós, nenhuma requisição será gerada. Caso contrário, o valor encontrado na matriz $\Lambda$ será multiplicado pelo percentual de carga. Para maximizar os efeitos da carga de tráfego, a distribuição de chegada de pacotes adotada foi a Constant Bit Rate. Os instantes iniciais e duração de cada requisição ocorrem de forma aleatória.

Em complemento, tanto no processo de VTD quanto na simulação foi empregada a mesma matriz de demanda de tráfego para todas as topologias em todas as classes.

Nesse trabalho são confrontadas estatísticas de congestionamento, probabilidade de bloqueio e utilização média dos enlaces [5]. Nesse contexto, probabilidade de bloqueio é a relação entre o número de requisições de caminhos ópticos bloqueados e o número total de requisições. Uma requisição será bloqueada se ela não puder ser acomodada em nenhum comprimento de onda. Já a utilização média dos enlaces é obtida calculando-se a média entre as porcentagens de uso efetivo da capacidade de transmissão em cada enlace. Para efeito de comparação, o congestionamento foi normalizado entre 0 e 1 , com o zero correspondendo ao menor valor encontrado, e o 1 ao maior.

A Figura 3 mostra a evolução do congestionamento normalizado em função do tamanho do anel para $H_{1}$ e $H_{0}$. O congestionamento na hierarquia superior apresenta um mínimo para três anéis. Isso pode ser facilmente explicado pelo fato de que três anéis formam um grafo completo [7]. O congestionamento na hierarquia inferior cai assintoticamente à medida que o número de anéis aumenta. 


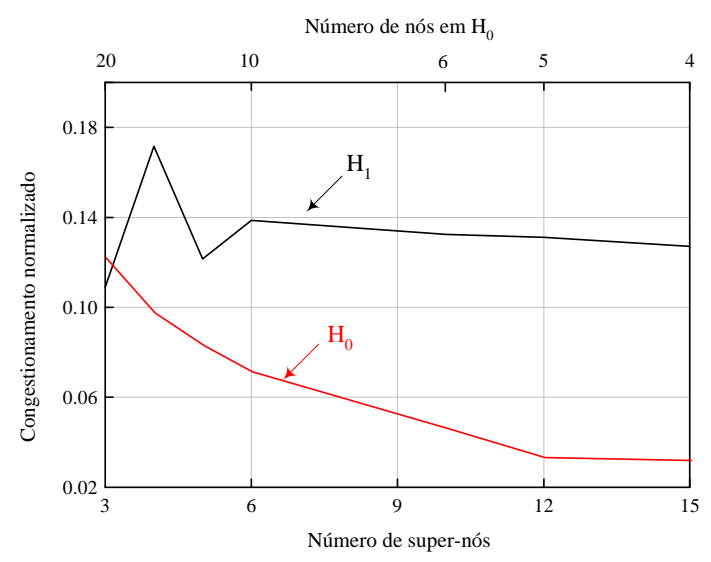

Fig. 3. Congestionamento normalizado para as hierarquias superior e inferior em função do número de super-nós em uma rede de 60 nós.

A Figura 4 mostra os resultados da simulação das sete redes de 60 nós em termos da carga na rede. Cada uma das redes escolhidas, para cada um dos níveis de carga, foi submetida ao OWns várias vezes, até serem obtidos 25 resultados. Os pontos que geram as curvas mostradas na Figura 4 resultam da média aritmética desses valores, sendo probabilidade de bloqueio na Figura 4(a) e utilização média dos enlaces na Figura 4(b). Ressaltamos que as redes com 12 e 15 supernós foram submetidas ao OWns mais vezes que as outras, a fim de obter o mesmo número de resultados, devido a um problema de uso de memória acusado com frequiência pelo OWns, conforme [3].

Um resultado importante observado na Figura 4(a) é um decréscimo de $6 \%$ da taxa de bloqueio quando o número de super-nós aumenta de 3 para 15. À medida que a carga na rede diminui, essa diferença também diminui.

Com relação à carga, a taxa de utilização da rede permanece praticamente constante para redes com 3, 4, 5 e 6 super-nós. Para redes com um número maior de super-nós ocorre uma variação maior.

Um fator associado ao custo financeiro da rede é o número de pares de tranceptores ópticos necessários para implementar uma dada topologia. A Figura 5 ilustra a variação desse número em função do número de super-nós na rede. Percebese o aumento linear do número de pares de tranceptores quando a rede utiliza um número maior de super-nós. Esse aumento deve-se ao maior número de comprimentos de ondas necessários na hierarquia superior.

Com base nos resultados aqui apresentados, obtemos as seguintes concusões:

1) Fixado $N$, quando analisamos uma rede com um determinado número de super-nós, a probabilidade de bloqueio tem variações pequenas com relação à carga da rede, com valores sempre inferiores a $7 \%$;

2) A utilização média dos enlaces da rede diminui à medida que aumentamos o número de super-nós na rede. Isso significa que boa parte dos recursos da rede serão sub utilizados. Esse efeito gera duas conseqüências diretas: o mal uso dos recursos da rede e a possibilidade da utilização desses recursos ociosos para o projeto de redes

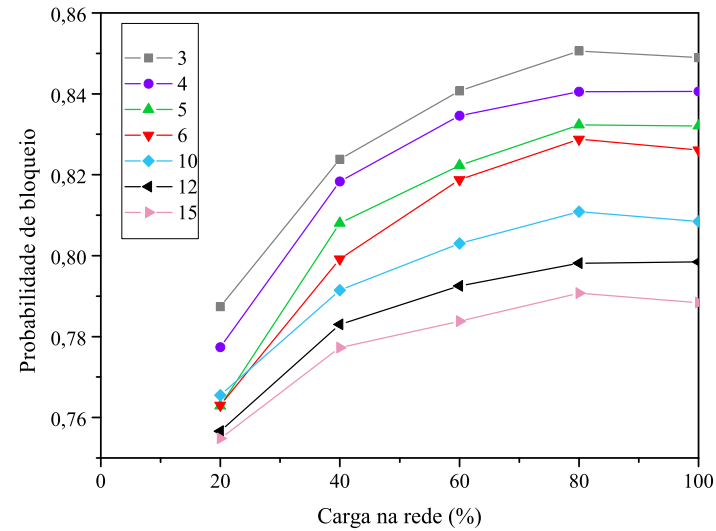

(a)

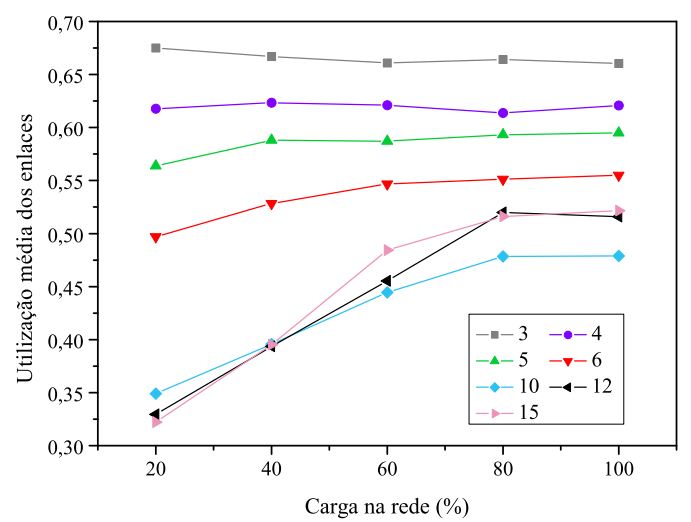

(b)

Fig. 4. (a) Probabilidade de bloqueio e (b) Utilização média dos enlaces em função da carga utilizada para a rede de 60 nós tendo como parâmetro o número de super-nós.

tolerantes à falhas;

3) O congestionamento não pode ser usado isoladamente para definir qual é a melhor topologia em projetos de redes ópticas. Quando confrontamos as Figuras 3 e 5 notamos que a configuração de 15 super-nós é levemente melhor que a de 12 super-nós, ao passo que o custo da última é consideravelmente menor. À medida que diminuímos o número de super-nós, o custo diminui linearmente, entretanto o congestionamento aumenta de forma expressiva. A decisão, portanto, de qual é a melhor configuração dependerá do contexto do projeto;

4) A probabilidade de bloqueio não pode ser usada isoladamente para definir qual é a melhor topologia em projetos de redes ópticas. Observando as Figuras 4(a) e 5, vemos que, quanto maior o número de super-nós, menor é a probabilidade de bloqueio, independentemente da carga posta sobre a rede. No entanto, o custo cresce para 


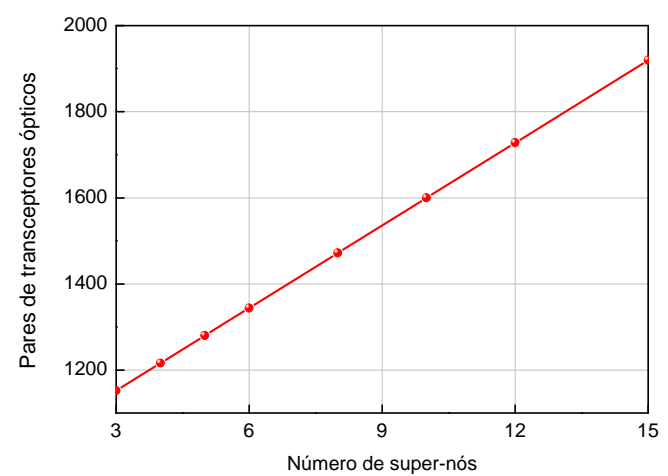

Fig. 5. Número de pares de transceptores ópticos em uma rede em anéis hierárquicos de 60 nós em função do número de super-nós.

redes com maior número de super-nós. Mais uma vez será preciso estabelecer um compromisso entre custo e métrica de desempenho. O acréscimo de $6 \%$ na taxa de bloqueio quando o número de super-nós diminui de 15 para 3 pode não ser preocupante em determinados contextos. Nesse caso, é preferível usar redes com menor número de super-nós, por possuírem custos menores.

\section{Conclusões}

Neste trabalho, apresentamos uma metodologia para o projeto de redes ópticas em anéis hierárquicos. Partindo da matriz de demanda de tráfego entre os nós, escolhe-se uma topologia virtual que apresente baixo valor de congestionamento. Usase esta topologia como entrada para o processo de roteamento de tráfego e alocação de comprimentos de onda, assumindo-se que ela pode ser mapeada fisicamente. Finalmente, analisa-se o desempenho da rede com o simulador de redes ópticas OWns.

Para estudo de caso escolhemos redes com 60 nós. Usamos um algoritmo de busca guloso para gerar topologias virtuais com $3,4,5,6,10,12$ ou 15 super-nós, e selecionar sete delas, uma de cada classe, observando o congestionamento. Para o roteamento e a alocação de comprimentos de onda, usamos algoritmos disponibilizados pelo software OWns. Os resultados foram apresentados em termos de congestionamento, probabilidade de bloqueio das requisições de tráfego e ocupação média da rede. O custo de cada solução encontrada pela metodologia aqui apresentada pode ser avaliado em termos do número de pares de tranceptores ópticos necessários para a construção da rede. A partir dos resultados obtidos com as redes de 60 nós, verificamos que tanto o congestionamento quanto a probabilidade de bloqueio não podem ser usados isoladamente para definir qual é a melhor topologia em projetos de redes ópticas.

Pretendemos no futuro estender a metodologia aqui apresentada para redes hierárquicas em malha. Vários critérios para o agrupamento de nós estão sendo estudados e devem ser apresentados em breve.

\section{AgRAdecimentos}

Os autores são gratos ao Prof. Antônio A. Belém e ao Sr. Rafael P. Esteves pelo auxílio com o OWns. Esse trabalho foi parcialmente financiado pela CAPES e pelo CNPq.

\section{REFERÊNCIAS}

[1] Mapa do Backbone RNP. Disponível em http://www.rnp.br/backbone/index.php. Acesso em 01/05/2007.

[2] NS-2 Network Simulator. Disponivel em http://www.isi.edu/nsnam/ns. Acesso em 01/05/2007.

[3] OWNS Readme. DAWN Networking Research Lab. Disponível em http://dawn.cs.umbc.edu/owns/obs_readme.html. Acesso em 30/07/2007.

[4] R. T. R. Almeida, L. C. Calmon, E. Oliveira, and M. E. V. Segatto. Design of virtual topologies for large optical networks through an efficient MILP formulation. Optical Switching and Networking, pages 2-10, 2006.

[5] D. Campelo, R. C. Almeida, and H. Waldman. Blocking probabilities generated by the first fit wavelength assignment algorithm in WDM rings. In III Workshop TIDIA, pages 60-62, Nov. 2006.

[6] R. M. Krishnaswamy and K. N. Sivarajan. Design of logical topologies: A linear formulation for wavelength-routed optical networks with no wavelength changers. IEEE/ACM Trans. Networking, 9:186-198, Apr. 2001

[7] C. Maioli, T. Botelho, M. Oliveira, L. Pereira, R. T. R. Almeida, M. R. N. Ribeiro, E. Oliveira, and M. E. V. Segatto. The design of hierarchical self-healing rings networks. In Proceedings of ConfTele'05, pages 5-8, Tomar-Portugal, Apr. 2005.

[8] B. Mukherjee. Optical Communication Networks. McGraw-Hill, 1997.

[9] E. Oliveira, L. C. B. Pereira, R. T. R. Almeida, and M. E. V. Segatto. A hibrid-combined algorithm approach for the design topologies and flow congestion minimization of optical networks. In Proceedings of ConfTele'05, pages 1-4, Tomar-Portugal, Apr. 2005.

[10] E. Oliveira, M. E. V. Segatto, M. M. O. Silva, F. R. de Souza, M. H. M. Paiva, G. Lavagnoli, M. O. Lima, R. T. R. Almeida, and A. S. Garcia. On solving HSHR networks. In Proceedings of ConfTele'07, PenichePortugal, May 2007.

[11] M. Pallu. Algoritmo para roteamento de sinais em redes ópticas. Master's thesis, Univ. Tec. Federal do Paraná, 2004.

[12] N. Parnis and E. V. Jones. Design of an optical pan-european transport network based on hierarchical rings. In IEEE Conference on Telecommunications, number 451, pages 206-210, Apr. 1998.

[13] L. C. B. Pereira, M. E. V. Segatto, R. T. R. Almeida, and E. Oliveira. Anéis lógicos disjuntos para projeto de topologias virtuais de redes ópticas tolerantes à falhas. In MOMAG04: XI Simpósio Brasileiro de Microondas e Optoeletrônica e VI Congresso Brasileiro de Eletromagnetismo, number 1, pages 22-27, Aug. 2004.

[14] R. Ramaswami and K. N. Sivarajan. Optical networks: A practical perspective. Morgan Kaufmann Publishers, Inc, San Francisco, 2001.

[15] J. Shi and J. P. Fonseka. Hierarchical self-healing rings. IEEE/ACM Trans. Networking, 3:690-697, Dec. 1995.

[16] J. Shi and J. P. Fonseka. Interconnection of self-healing rings. In IEEE, editor, ICC 96 - IEEE International Conference on Converging Technologies for Tomorrow's Applications, number 3, pages 1563-1567, June 1996.

[17] K. Sivalingam and B. Wen. Optical WDM network simulator (OWns): Architecture and performance studies. Technical report, School of Electrical Engineering and Computer Science. Washington State University, 2001.

[18] Y. Xin, G. N. Rouskas, and H. G. Perros. On the physical and logical topology design of large-scale optical networks. IEEE J. Lightwave Technology, 21(4):904-915, Apr. 2003.

[19] A. Zalesky, H. L. Vu, M. Zukerman, and I. Ouveysi. A framework for solving logical topology design problems within constrained computation time. IEEE Communication Letters, 7(10), Oct. 2003. 